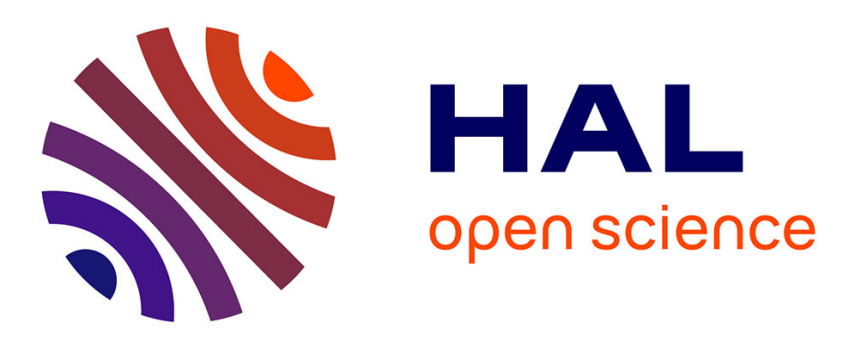

\title{
Agent-Based Modeling Using Swarm Intelligence in Geographical Information Systems
}

Rawan Ghnemat, Cyrille Bertelle, Gérard H.E. Duchamp

\section{To cite this version:}

Rawan Ghnemat, Cyrille Bertelle, Gérard H.E. Duchamp. Agent-Based Modeling Using Swarm Intelligence in Geographical Information Systems. Innovations'08, Dec 2008, Al-Ain, United Arab Emirates. pp.xxx-xxx. hal-00430564

\section{HAL Id: hal-00430564 https://hal.science/hal-00430564}

Submitted on 9 Nov 2009

HAL is a multi-disciplinary open access archive for the deposit and dissemination of scientific research documents, whether they are published or not. The documents may come from teaching and research institutions in France or abroad, or from public or private research centers.
L'archive ouverte pluridisciplinaire HAL, est destinée au dépôt et à la diffusion de documents scientifiques de niveau recherche, publiés ou non, émanant des établissements d'enseignement et de recherche français ou étrangers, des laboratoires publics ou privés. 


\section{Agent-Based Modeling Using Swarm Intelligence in Geographical Information Systems}

\author{
Rawan Ghnemat, Cyrille Bertelle \\ LITIS - University of Le Havre \\ 25 rue Philippe Lebon - BP 540 \\ 76058 Le Havre cedex, France
}

\author{
Gérard H.E. Duchamp \\ LIPN - University of Paris XIII \\ 99 avenue Jean-Baptiste Clément \\ 93430 Villetaneuse, France
}

\begin{abstract}
In this paper swarm intelligence algorithms are presented to deal with dynamical and spatial Organization emergence. The goal is to model and simulate the development of spatial center and their dynamic interactions with the environment and the individuals; the swarm algorithms used are inspired from natural termite nest building and Ant culturing algorithm. Combination of decentralized approaches based on emergent clustering mixed with spatial multi criteria constraints or attractions developed, extension of termite nest building algorithms has been proposed to have multi center adaptive process. The modeling has been made using agent based modeling techniques and the simulation developed using Repast (REcursive Porous Agent Simulation Toolkit) and OpenMap as geographical information system (GIS) software, some simulations result are provided.
\end{abstract}

\section{INTRODUCTION}

Many natural and artificial systems have emergent properties based on spatial development. This spatial development is both the result of some mecanisms from the system behavior and the actor of the system formation by morphogenetic feedbacks [2]. Natural ecosystems or urban dynamics are typically based on systems which evolve mainly under the influence of such spatial emergent organizations. The goal of this paper is to study some models and algorithms able to describe such spatial selforganization processes, taking into account the complexity of the phenomena. Decision makers, like urban planners, need a better understanding of such a dynamics in order to adapt the infrastructure development according to the feed-back of citizen use. Simulation allows analysis and predictive studies.
Benenson [1] proposes to classify urban modelling in two classes: macro-modelling and individual-based modelling. The first one fails in accurate individual and emergent phenomena modelling. In the second one, cellular automata are often used to describe rule-based systems for diffusion phenomena description on regular grid space. The proposed model, based on swarm intelligence, allows to model dynamical emergence on more general spaces described by geographical information system.

In section 2, complex systems concepts are defined and their applications to urban dynamics understanding are described, then swarm intelligence algorithms are described as methodologies to implement the complex systems concepts, using distributed computations. In section 3, we propose some specific swarm intelligence methods based on ant systems in order to model the spatial organizations emergence and we give some experiments using RePast multi-agent platform mixed with a geographical information system.

\section{MODELLING SPATIAL COMPLEXITY}

\subsection{Complex System Concepts}

Complex system theory [6] is based on the fact that for many applicative domains, we can find similar processes linking emergent global behavior and interaction network of constituents. The global behavior is generally not accessible using classical analytical methods.

In classical analytical methods, the global behavior of the system is the description of the equations. Simulations from these formulations, consist in obtaining the trajectories of the behavior predefined in the equation formulation.

In complex systems modelling, we have to model the constituents of the system and the interaction network or system which links these constituents, using a decentralized 


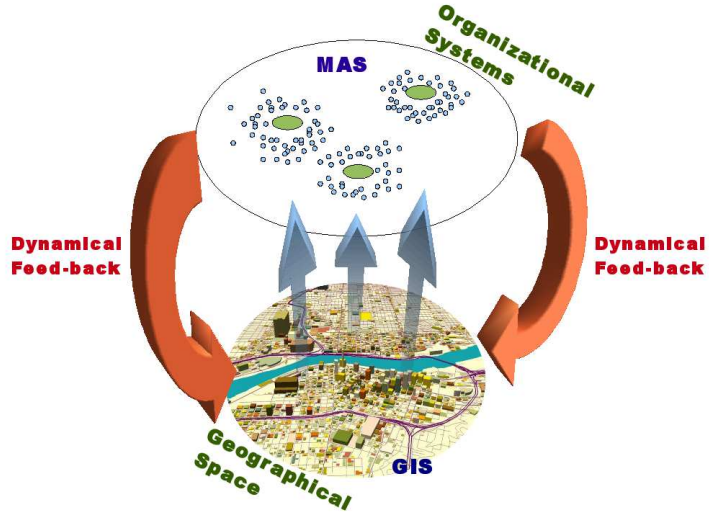

Figure 1. Spatial organizations Complex-
ity Description and the Conceptual Generic
Model Based on Swarm Intelligence

approach. So the global behavior of the system cannot be understood by the description of each constituent. In complex systems modelling, the global behavior is an emergent property from the interaction network or systems between its constituents and leads to the creation of an dynamical organization of these constituents

This dynamical and emergent organization retro-acts on its own components. In many natural and artificial systems, the environement has some spatial effects which interact on the whole complexity of the phenomenon. This spatial environment can be modified by the system but he can also be the catalyst of its own evolution. Understanding and modelling the deep structural effect of the interaction between the systems and its spatial environment is the goal of the study presented in the sequel.

\subsection{Application to urban dynamics}

Social and human developments are typical complex systems. Urban development and dynamics are the perfect illustration of systems where spatial emergence, selforganization and structural interaction between the system and its components occur. In Figure 1, we concentrate on the emergence of organizational systems from geographical systems. The continuous dynamic development of the organization feed-back on the geographical system which contains the organization components and their environment. For the organization detection, we use swarm intelligence processes. We model the feed-back process of this emergent organization on the system constituents and its environment. To analyse or simulate urban dynamics, nowadays, we can use the great amount of geographical databases directly available for computational treatment within Geographical Information Systems. On the organizational level description, the new development of multiagent systems (MAS) allows nowadays to develop suitable models and efficient simulations.

The applications we focus on in the models that we will propose in the following concerns specifically the multi-center (or multi-organizational) phenomona inside urban development. As an artificial ecosystem, the city development has to deal with many challenges, specifically for sustainable development, mixing economical, social and environmental aspects. The decentralized methodology proposed in the following allows to deal with multi-criteria problems, leading to propose a decision making assistance, based on simulation analysis.

Cultural dynamics processes in urban area can be modelled using such a methodology. It is typically a multicriteria self-organization process where appears emergent usage of urban equipment. A modelling of these dynamics is presented latter in this paper.

\subsection{Swarm Intelligence and Spatial Envi- ronment}

Decentralized algorithms have been implemented for many years for various purposes. In this algorithm category, multi-agent systems can be considered as generic methods [10]. We deal, in this paper, with agents based on reactive architecture and expected to be used inside numerous entity-based systems. The aim of programs using such architectures, is to deal with emergent organizations using specific algorithms called emergent computing algorithms. Swarm Intelligence is the terminology used to point out such reactive agent-based methods where each entity is built with the same basis of behavior, but reacts in an autonoumous way.

Based on swarm intelligence concepts, Ant systems [3] propose some bio-inspired method from social insects mecanisms. For example, Ant Colony Optimization (ACO) methods is such a family of methods where the basic entities are virtual ants which cooperate to find the solution of graph-based problems, like network routing problems, for example. Using indirect communications, based on pheromon deposites over the environment (here a graph), the virtual ants react in elementary way by a probabilistic choice of path weighted with two coefficients, one comes from the problem heuristic and the other represents the pheromon rate deposit by all the ants until now. The feed-back process of the whole system over the entities is 
modelled by the pheromon action on the ants themselves.

Particule Swarm Optimization (PSO) is a metaheuristic method initially proposed by J. Kennedy and R. Ebenhart [5] which is also based on swarm intelligence concepts. This method is initialized with a virtual particle set which can move over the space of solutions corresponding to a specific optimization problem. The method can be considered as an extension of a bird flocking model, like the BOIDS simulation from C.W. Reynolds [8]. In PSO algorithm, each virtual particle moves according to its current velocity, its best previous position and the best position obtained from the particles of its neighborhood. The feed-back process of the whole system over the entities is modelled by the storage of this two best positions as the result of communications between the system entities.

Other swarm optimization methods have been developped like Artificial Immune Systems [4] which is based on the metaphor of an immune system as a collective intelligence process. F. Schweitzer proposes also a generic method based on distributed agents, using approaches of statistical many-particle physics [9].

The method proposed in this paper is based on Ant systems, allowing to deal with self-organization processes emerging from spatial constraints and attractive areas.

\section{MULTI-CENTER ANT NEST BUILDING TO MODEL EMERGENT SPATIAL OR- GANIZATIONS}

We will describe in this section, the general algorithm which is proposed to model emergent spatial organizations. This algorithm is based on the ant clustering. We introduce pheromon template to spatially control the clustering from local attraction. This method is a decentralized approach which allows to combinate multi-center and multi-criteria problems and we will show how we can apply it to model cultural dynamics in urban areas.

\subsection{Ant clustering}

Ant clustering algorithms are inspired by the corposes or larvea classification and aggregation that the ants colony are able to do in the real life. The ants are moving inside a closed area and are able to move some material which are randomly put on this area. After a while, and without any kind of centralized coordonation, the ants success to create some material clusters.

The algorithm is based on the following and very simple behavioral rules that each ant implements :
- When an ant is moving without carrying yet material and finds some material, the ant will take the material respecting the probability number :

$$
P_{p}=\left(\frac{k_{1}}{k_{1}+f}\right)^{2}
$$

where $f$ is the material density that the ant perceives locally around itself and $k_{1}$ is the treshold. It is easy to check that if $f<<k_{1}$ then $P_{p}$ is near the value 1 and if $f>>k_{1}$ then $P_{p}$ is near the value 0 .

- When an ant is moving when carrying some material, the probability to deposit it is computed by :

$$
P_{d}=\left(\frac{f}{k_{2}+f}\right)^{2}
$$

where $f$ is still the material density that the ant perceives locally around itself and $k_{2}$ is another treshold. It is easy to check that if $f<<k_{2}$ then $P_{d}$ is near the value 0 and if $f>>k_{2}$ then $P_{d}$ is near the value 1 .

\subsection{Spatial constraints using template}

The ant clustering shows some spatial self-organizations but has the specificity to generate clusters at random places. According to the first random moves that the ants starts to do in the beginning of the algorithm, some material will initiate aggregation and the clustering processus will complete this aggregation from these initial random first aggregations. To simulate some urban dynamics, we need to introduce specific location with respect to city center for example or cultural equipments. The clustering here will represent the people usage of these centers or equipments and we need to introduce an attractive effect by using a pheromon template. This method follows the algorithm known as Ant Nest Building [3]. In ant colonies, the center corresponds to the position of the queen which needs to build the nest and the ant colony moves around it to protect the nest by various material taken on the ground. The queen emits a pheromon which allows to attract the ants during their building. The ant has to deposit the material carried only if the pheromon quantity perceived belongs to a specific range. We use an attractive fonction called $P_{t}$, corresponding to a pheromon template and represented by the part (a) of the Figure 2.

Using this template function, we remplace in the clustering algorithm, the two provious probabilities defined in equation (1) and equation (2) by

$$
\begin{gathered}
P_{p}^{\prime}=P_{p}\left(1-P_{t}\right) \\
P_{d}^{\prime}=P_{d} P_{t}
\end{gathered}
$$




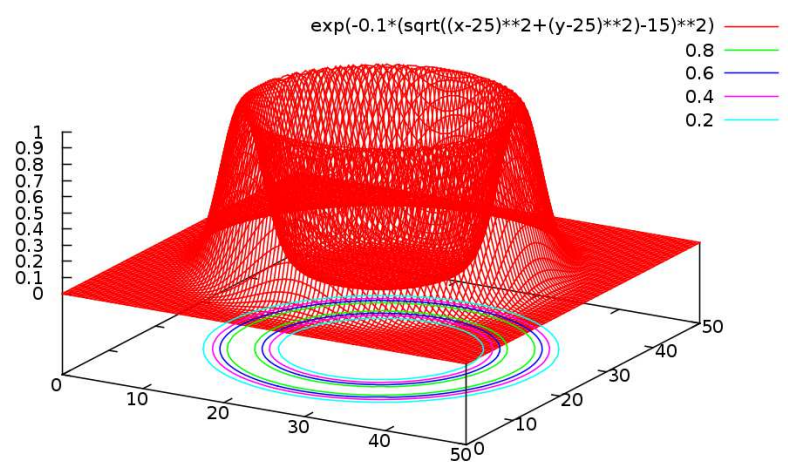

(a) Template function

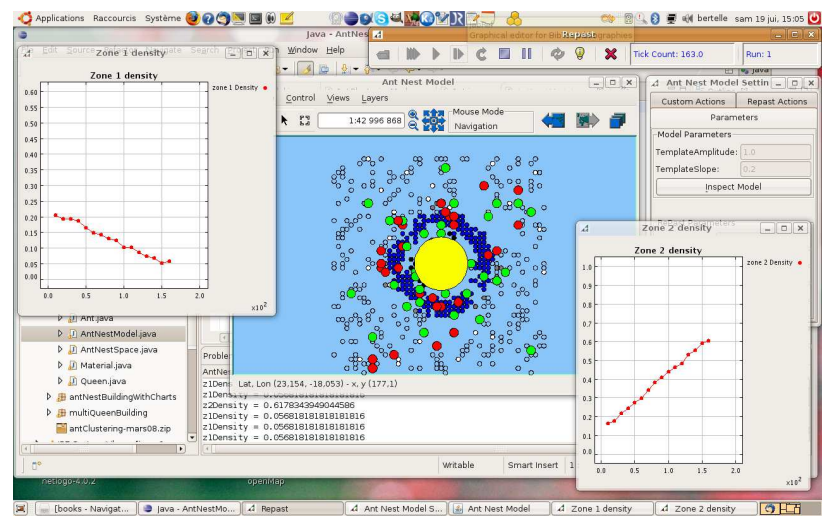

(b) Simulation on RePast

\section{Figure 2. Ant nest building with one center using RePast MAS paltform over OpenMap GIS}

In Figure 2, we show an implementation of this algorithm using the multi-agent platform called Repast [7]. The java version of this platform includes some packages allowing to interface with geographical databases and geographical information systems (GIS). The graphical output windows is made under OpenMap which is a GIS developped in Java. In this Figure, the materials moved by the ants are the small grey circles, the ants moving without material are the green circles, the ants carrying material are the red circles and the queen location is the yellow circle.

\subsection{Multi-template modelling}

The previous subsection describes one local attractive process characterized by the queen and its pheromon template emission. The advantage of this method is to be able to combine the solutions of multi-center and multi-criteria problems, using interactive processes, each

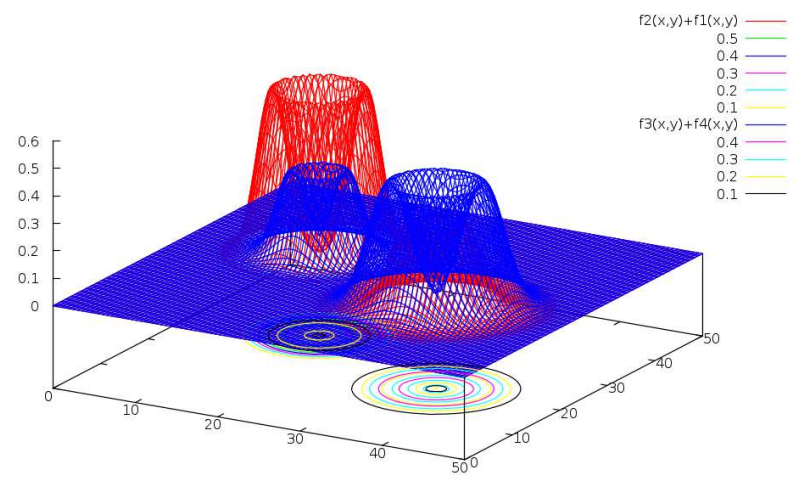

(a) Template functions

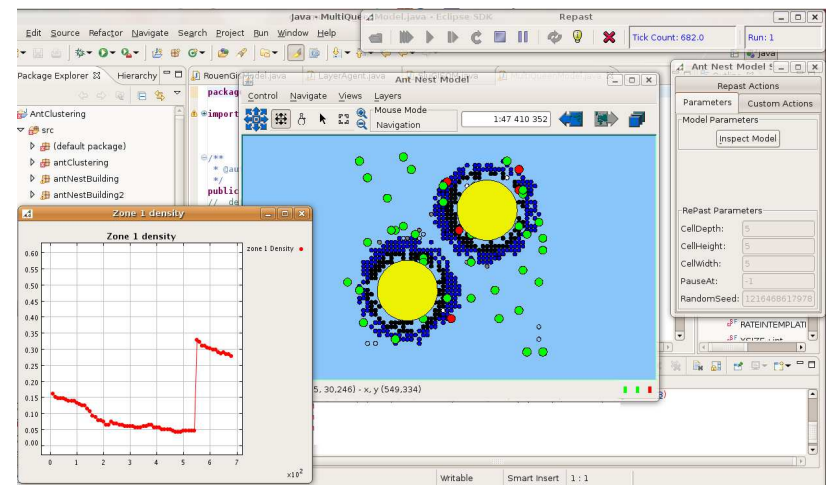

(b) Simulation on RePast

\section{Figure 3. Multi-center ant nest buiding using RePast over OpenMap}

one is represented by a queen and its pheromon template.

Figure 3 shows the simulation with two queens and two pheromon templates. It is possible also for each queen to emit many different kinds of pheromons: we called them colored pheromons. Each colored pheromon will attract only the ants associated to its color.

\subsection{Application to cultural equipment dy- namics}

The multi-template modelling can be used to model cultural equipment dynamics as described in Figure 4. On this Figure, we associate to each cultural center (cinema, theatre, ...) a queen. Each queen will emit many pheromon templates, each template is associated to a specific criterium (according to age, sex, ...). Initially, we put the material in the residential place. Each material has some characteristics, corresponding to the people living in this residential area. The simulation shows the spatial organization proces- 


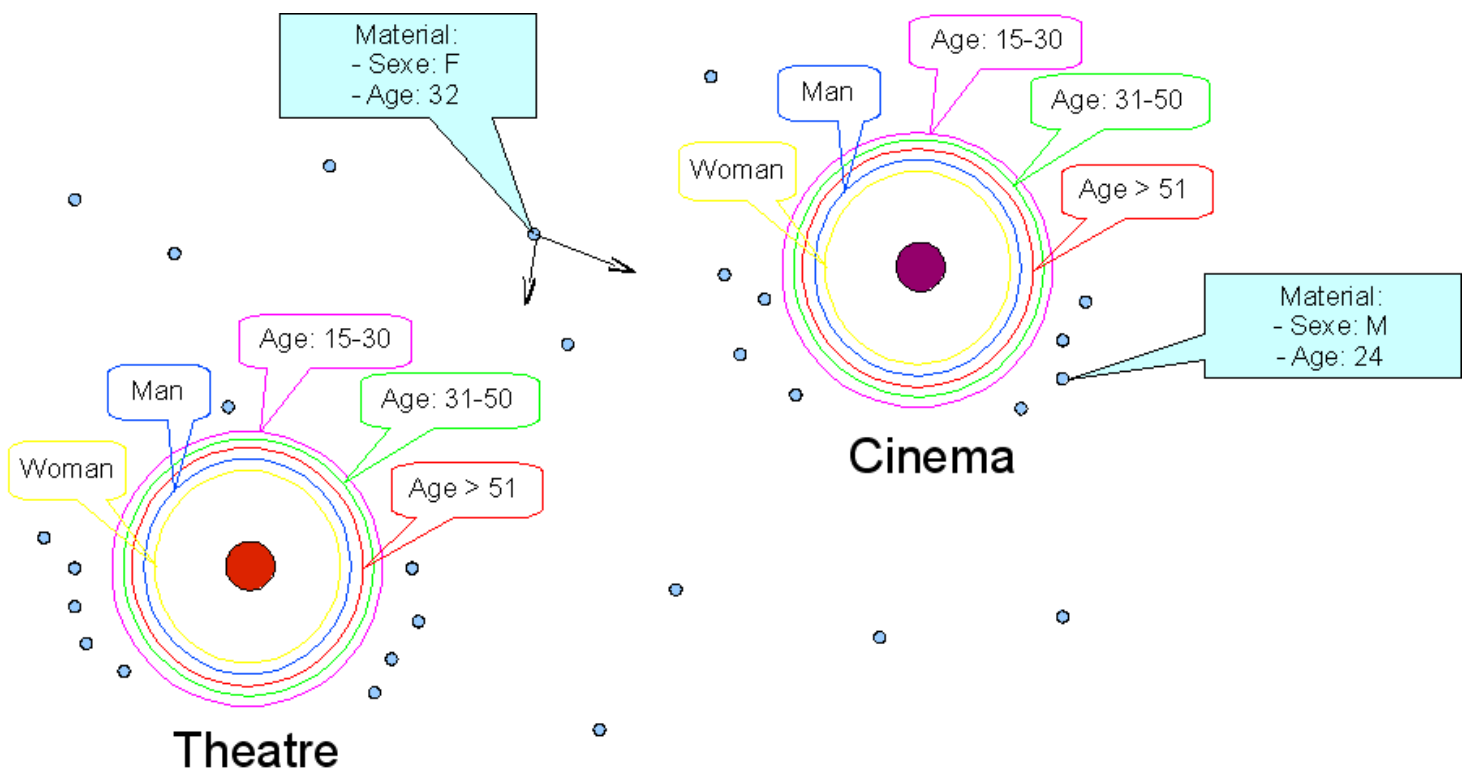

Figure 4. Cultural Equipment Dynamics Modelling

sus as the result of the set of the attractive effect of all the center and all the templates.

\section{Conclusion and Perspectives}

The paper develops some specific swarm intelligence algorithms based on ant colonies processes. Using such decentralized methods, we can model complex multi-center and multi-criteria self-organizations. Urban dynamics are one of the most relevant problems where these approaches can be efficient. This method can be used for any kind of urban infrastructure where the mechanism of using this infrastructure is complex like telecommunication infrastructures for example.

\section{References}

[1] Benenson, I. (1999) Modeling population dynamics in the city: from a regional to a multi-agent approach. Discrete Dynamics in Nature ans Society, 3:149-170.

[2] Bertelle, C.; Duchamp, G.H.E.; Kadri-Dahmani, H. (eds), Complex Systems and Self-Organization Modelling, "Understanding Complex Systems" series, Springer-Verlag, 2009 (in press).

[3] Bonabeau, E.; Dorigo, M.; Theraulaz, G. (1999) Swarm Intelligence, from natural to artificial systems, a volume in the Santa Fe Institute Studies in the Sciences of Complexity, Oxford University Press.
[4] De Castro, L.N.; Timmis, J. (2002) Artificial immune system: a new computational approach, SpringerVerlag, London, U.K.

[5] Kennedy, J.; Eberhart, R.C. (1995) Particle Swarm Optimization In Proceedings of IEEE International Conference on Neural Networks (Perth, Australia), IEEE Service Center, Piscataway, NJ, 5(3), pp 1942-1948.

[6] Le Moigne, J.-L. (1999) La modélisation des systèmes complexes, Dunod.

[7] Repast web site (2008) http://repast. sourceforge.net.

[8] Reynolds, C.W. (1987) Flocks, Herds and Schools: a distributed behavioral model In Computer Graphics, 21(4) (SIGGRAPH'87 Conference Proceedings), pp 25-34.

[9] Schweitzer, F. (2003) Brownian Agents and Active Particles, Springer.

[10] Weiss, G. (ed.) (1999) Multiagent Systems, MIT Press. 